\title{
Interprétation des profils aux interfaces dans l'analyse par SIMS des multicouches métalliques
}

\author{
O. Nemraoui*, M. Miloche*, M. Kaabouchi*,** et C. Sella* \\ * Laboratoire de Magnétisme et d'Optique, CNRS, 1 place Aristide Briand, 92195 Meudon, France \\ ** Faculté des Sciences, Département de Physique, Laboratoire de Physique des Solides, BP. 1796, Fès \\ Atlas, Maroc
}

\begin{abstract}
Résumé : L'analyse des interfaces dans les multicouches métalliques à partir des profils SIMS obtenus en utilisant un faisceau primaire d'ions $\mathrm{Cs}^{+}$et en détectant les ions moléculaires $\mathrm{MCs}^{+}$( $\mathrm{M}$ étant l'élément à analyser) montre que dans de nombreux cas on observe une variation anormale du rendement ionique de $\mathrm{MCs}^{+}$aux interfaces. Ce phénomène est lié d'une part à l'existence d'une couche interfaciale d'interdiffusion d'épaisseur nanométrique et d'autre part au rapport de densité des deux métaux constituant la multicouche. Quand le rapport de densité du métal lourd à celle du métal léger est inférieur à 1,4 cet anomalie de rendement ionique aux interfaces n'apparaît pas. C'est le cas des multicouches $\mathrm{Ni}-\mathrm{Fe}, \mathrm{Co}-\mathrm{Fe}, \mathrm{Cu}-\mathrm{Co}, \mathrm{Nb}-\mathrm{Fe}, \mathrm{Fe}-\mathrm{V}, \mathrm{Ag}-\mathrm{Co}, \mathrm{Co}-\mathrm{Zr}, \mathrm{V}-\mathrm{Ti}$, Al-Si pour lesquelles ce rapport varie de 1,13 à 1,36. Quand ce rapport est supérieur à 1,4 on observe une forte exaltation du signal $\mathrm{MCs}^{+}$de l'élément léger et en général une atténuation du signal de l'élément lourd. C'est le cas des multicouches $\mathrm{Ni}-\mathrm{V}$, Ti-Al, Fe-Ti, Nb-Ti, Co-Ti, Ni-Ti, Cu-Ti, Cl-Al, Ni-Al, W-Ti, W-Al, Pt-Al, Pt-Si pour lesquelles ce rapport varie de 1,46 à 9,18 . Ces résultats ont été confirmés par une étude comparative des intensités SIMS données par chacun des deux métaux à l'état pur et à l'état d'alliages homogènes de différentes compositions obtenues en couches minces par copulvérisation.
\end{abstract}

\section{INTRODUCTION}

Les multicouches métalliques déposées sur substrats superpolis (verre ou silicium) sont bien adaptées à l'évaluation de la résolution en profondeur des profils SIMS et à l'étude de la qualité des interfaces (planéité, interdiffusion, degré de contamination...). On utilise pour cela un faisceau primaire d'ions $\mathrm{Cs}^{+}$et on analyse les ions moléculaires $\mathrm{MCs}^{+}$( $\mathrm{M}$ est l'élément à analyser), ce qui permet en principe de réduire les effets de matrice [1]. Cependant dans de nombreux cas on observe une forte variation du rendement ionique de $\mathrm{MCs}^{+}$aux interfaces.

Dans un travail consacré aux multicouches $\mathrm{Ni}-\mathrm{Ti}$ [2] une forte exaltation du signal $\mathrm{TiCs}^{+}$avait été observée aux interfaces. Cet effet avait été associé à l'existence d'une couche interfaciale d'interdiffusion d'épaisseur nanométrique $(5$ à $15 \AA ̊)$. Cette couche interfaciale avait été révélée et étudiée au moyen de différentes techniques (diffraction électronique, magnétometrie, reflectometrie de rayons $X$ ou de neutrons sous incidence rasante) [3], l'épaisseur de cette couche d'interdiffusion étant reliée aux conditions de dépôt (température du substrat, durée de dépôt, etc...). L'exaltation au niveau de cette couche de diffusion intefaciale avait été confirmée par une étude de la comparaison des rendements d'ions secondaires $\mathrm{MCs}^{+}$ dans des couches homogènes d'alliages NiTi de différentes compositions qui donnaient lieu également a une forte exaltation du rendement de $\mathrm{TiCs}^{+}[2,4]$. Des exaltations similaires de $\mathrm{TiCs}^{+}$avait été observé au niveau des interfaces dans les multicouches $\mathrm{Co}$ - $\mathrm{Ti}$ et $\mathrm{Fe}-\mathrm{Ti}$ [2] alors qu'aucun effet d'exaltation n'apparaissait dans les systèmes $\mathrm{Fe}-\mathrm{Co}, \mathrm{Fe}-\mathrm{Ni}$, $\mathrm{Co}-\mathrm{Ni}$. Or dans ces trois derniers cas l'étude microstructurale et les mesures magnétiques indiquaient l'existence d'une couche d'interdiffusion d'épaisseur nanométrique. La présence d'une couche d'interdiffusion semble donc nécessaire mais pas suffisante pour donner lieu à l'effet d'exaltation.

Pour analyser les différents paramètres physiques associés à ce phénomène une étude systématique a été entreprise sur une série de multicouches métalliques (Al-Ti, Al-Nb, Ni-Ti, Fe-Ti, Co-Ti, Nb-Ti, V-Ti, W-Ti, Co-Ag, Co-Cu, Co-Pt, Fe-Co, Fe-Ni, Fe-Nb, Fe-V, Ni-V, Pt-Si, Pt-Al, Al-Si). 


\section{TECHNIQUES EXPERIMENTALES}

Les différentes multicouches ont été déposées par pulvérisation cathodique en utilisant un système triode continu [5]. Les paramètres de dépôt sont contrôlés par ordinateur ce qui permet d'obtenir une très grande régularité des empilements avec des interfaces abruptes. Par ailleurs des films d'alliages métalliques binaires ont été déposés par la technique de la copulverisation radiofréquence. Ces films sont ensuite recouverts de deux couches des métaux purs constituant l'alliage. Cet empilement tricouche permet une comparaison du rendement ionique des deux métaux purs et de ces même métaux dans un alliage binaire. Les composition des films d'alliages déposés ont été déterminées au moyen de la microsonde électronique. Les profils SIMS ont été réalisés sur la microsonde ionique Cameca $\mathrm{MS}-3 \mathrm{~F}$ en utilisant un faisceau primaire d'ions $\mathrm{Cs}^{+}$de $5.5 \mathrm{KeV}$ avec un angle d'incidence de $42^{\circ}$ et un courant de $10 \mathrm{nA}$, et en détectant les ions moléculaires $\mathrm{MCs}^{+}$. Les conditions d'érosion ont été choisies pour minimiser la rugosité induite par l'attaque ionique et reduire le phénomène de mixing au niveau des interfaces. Typiquement la rugosité initiale de la plupart des multicouches mesurée au profilometre Tencor se situait dans la gamme de 7-10 $\AA$ alors que dans le fond du cratère une rugosité moyenne de 10 à $15 \AA ̊$ a été observée.

\section{RESULTATS ET DISCUSSION}

La comparaison des résultats expérimentaux et une évaluation critique des différents paramètres physiques des échantillons étudiés font apparaître une corrélation entre le phénomène d'exaltation aux interfaces et le rapport des densités des deux métaux constituant la multicouche. En effet quand le rapport de densité de

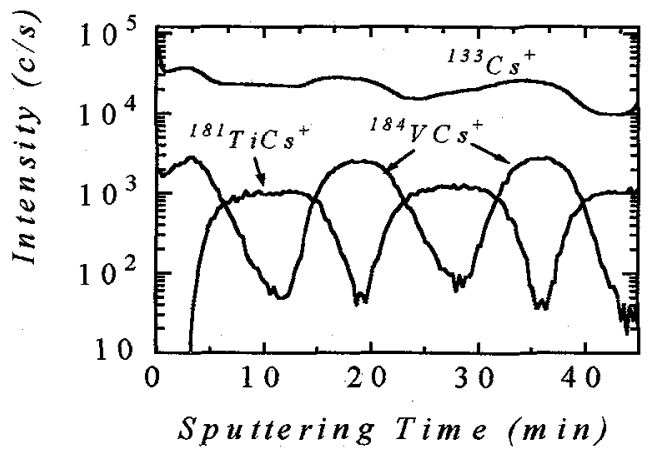

Figure 1: Profil SIMS d'une multicouche V-Ti $\left[\mathrm{e}_{\mathrm{V}}=250 \AA ; \mathrm{e}_{\mathrm{Ti}}=300 \AA\right]$

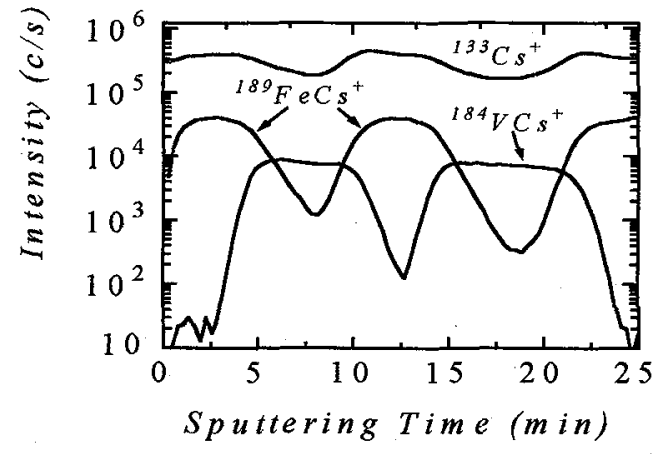

Figure 2 : Profil SIMS d'une multicouche Fe-V $\left[\mathrm{e}_{\mathrm{V}}=\mathrm{e}_{\mathrm{re}}=300 \AA\right]$

l'élément lourd à celle de l'élément léger est inférieur à 1,4 cette anomalie de rendement ionique aux interfaces n'apparaît pas. C'est le cas des multicouches $\mathrm{Ni}-\mathrm{Fe}, \mathrm{Co}-\mathrm{Fe}, \mathrm{Cu}-\mathrm{Co}, \mathrm{Nb}-\mathrm{Fe}, \mathrm{Fe}-\mathrm{V}, \mathrm{Ag}-\mathrm{Co}, \mathrm{Co}-$ $\mathrm{Zr}, \mathrm{V}-\mathrm{Ti}$, Al-Si pour lesquelles ce rapport varie de 1,13 à 1,36. Ce type de profil est illustré par les figures 1,2 et 3 .

Quand ce rapport est supérieur à 1,4 on observe une forte exaltation du signal $\mathrm{MCs}^{+}$de l'élément léger et en général une atténuation du signal de l'élément lourd. C'est le cas des multicouches Ni-V, Ti-Al, Fe-Ti, $\mathrm{Nb}-\mathrm{Ti}, \mathrm{Co}-\mathrm{Ti}, \mathrm{Ni}-\mathrm{Ti}, \mathrm{Cu}-\mathrm{Ti}, \mathrm{Cu}-\mathrm{Al}, \mathrm{Ni}-\mathrm{Al}, \mathrm{W}-\mathrm{Ti}, \mathrm{W}-\mathrm{Al}, \mathrm{Pt}-\mathrm{Si}, \mathrm{Pt}-\mathrm{Al}$ pour lesquelles ce rapport varie de 1,46 à 9,18 . Ce comportement est illustré par les figures 4,5 et 6 . On remarque que le profil SIMS de la multicouche $\mathrm{Ni}-\mathrm{Ti}\left(\mathrm{d}_{\mathrm{Ni}} / \mathrm{d}_{\mathrm{Ti}}=1,97\right)$ (fig. 4) ainsi que celui de la multicouche $\mathrm{Ni}-\mathrm{V}\left(\mathrm{d}_{\mathrm{Ni}} / \mathrm{d}_{\mathrm{V}}=1,46\right)($ fig. 5) presentent respectivement une forte exaltation de $\mathrm{TiCs}^{+}$et de $\mathrm{VCs}^{+}$aux interfaces alors que le phénomène d'exaltation disparait dans le cas de la multicouche V-Ti (fig. 1) pour laquelle le rapport des densités $\left(d_{V} / d_{T 1}=1,35\right)$ est inferieur à 1,4 . La même remarque peut être faite dans la comparaison des systemes Fe$\mathrm{Ti}$ et $\mathrm{Fe}-\mathrm{V}$. Dans le cas des multicouches Fe-Ti (fig. 6) pour lesquelle $\mathrm{d}_{\mathrm{Fe}} / \mathrm{d}_{\mathrm{Ti}}=1,75$ le signal $\mathrm{TiCs}^{+}$est 


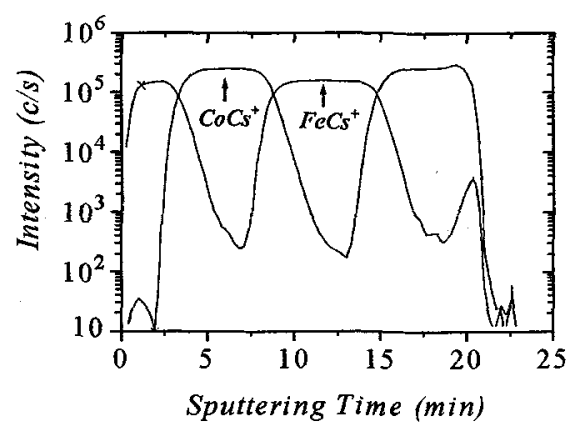

Figure 3 : Profil SIMS d'une multicouche $\mathrm{Co}-\mathrm{Fe}$ $\left[\mathrm{e}_{\mathrm{Co}}=\mathrm{e}_{\mathrm{Fe}}=300 \AA\right]$

fortement exalté aux interfaces alors que dans le cas du systeme $\mathrm{Fe}-\mathrm{V}$ pour lequel $\mathrm{d}_{\mathrm{Fe}} / \mathrm{d}_{\mathrm{V}}=1,3$ aucun effet d'exaltation n'est observé (fig. 2).

Si l'on associe maintenant au titane un métal plus léger comme l'aluminium c'est l'élément le plus léger qui donnera lieu au phénomène d'exaltation. Ainsi dans une bicouche Ti-Al $\left(\mathrm{d}_{\mathrm{T}} / \mathrm{d}_{\mathrm{Al}}=1,67\right)$ l'exaltation du signal $\mathrm{AlCs}^{+}$est observé à l'interface (fig.8). Ce résultat peut être comparé à l'exaltation de $\mathrm{TiCs}^{+}$dans les multicouches $\mathrm{Ni}-\mathrm{Ti}$ (fig.4), $\mathrm{Fe}-\mathrm{Ti}$ (fig. 6) et $\mathrm{Co}-\mathrm{Ti}$ (fig. 7). Dans les quatres exemples l'exaltation du signal $\mathrm{MCs}^{+}$se produit pour le métal le plus léger: $\mathrm{Al}$ dans le systeme Ti-Al et $\mathrm{Ti}$ dans les systemes $\mathrm{Fe}-\mathrm{Ti}$, $\mathrm{Ni}-\mathrm{Ti}$.et $\mathrm{Co}-\mathrm{Ti}$.

A ce stade de l'étude il apparait que l'effet d'exaltation est lié d'une part à l'existence d'une couche d'interdiffusion d'épaisseur nanométrique et d'autre part au rapport des densités des deux éléments constituant la multicouche.

On peut alors supposer que dans des alliages binaires homogènes formés à partir de ces deux élements, on observera le même phénomène d'exaltation du rendement ionique de l'ion $\mathrm{MCs}^{+}$correspondant à l'élément le plus léger.

Pour cela nous avons préparé des couches d'alliages binaires recouvertes de couches des deux métaux purs constituant l'alliage. Dans le cas du système $\mathrm{NiTi}$, les alliages sont préparés par copulverisation RF à partir d'une cible de titane sur laquelle est placé un nombre de disques de nickel calculé en fonction de la

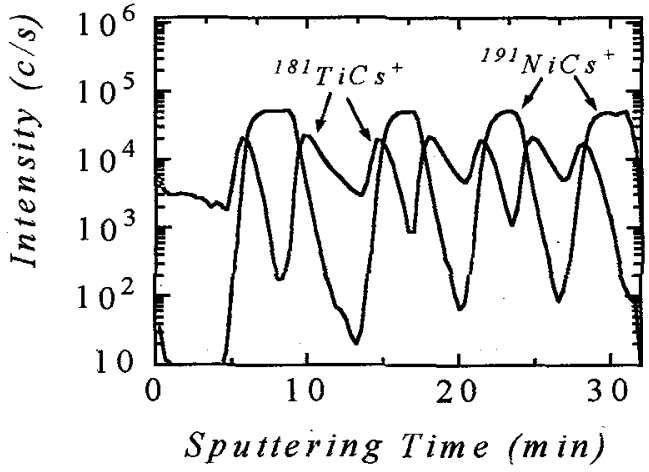

Figure 4 : Profil SIMS d'une multicouche Ni-Ti $\left[\mathrm{e}_{\mathrm{Ni}}=\mathrm{e}_{\mathrm{Ti}}=200 \AA\right]$

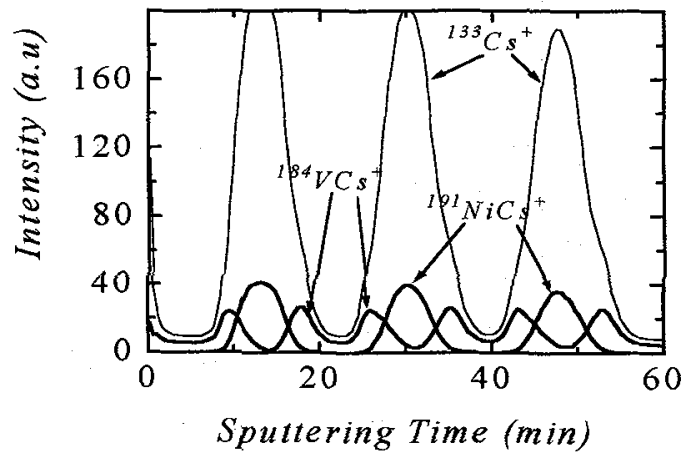

Figure 5 : Profil SIMS d'une multicouche Ni-V $\left[\mathrm{e}_{\mathrm{Ni}}=300 \AA ; \mathrm{e}_{\mathrm{V}}=200 \AA\right]$ 


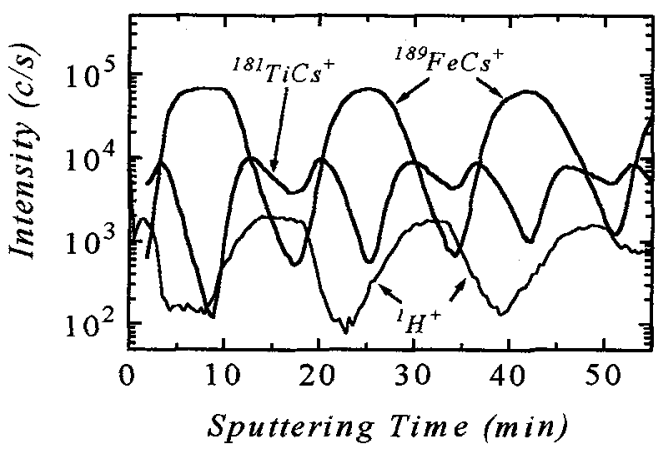

Figure 6: Profil SIMS d'une multicouche Fe-Ti $\left[\mathrm{e}_{\mathrm{Fe}}=300 \AA ; \mathrm{e}_{\mathrm{Ti}}=200 \AA\right]$

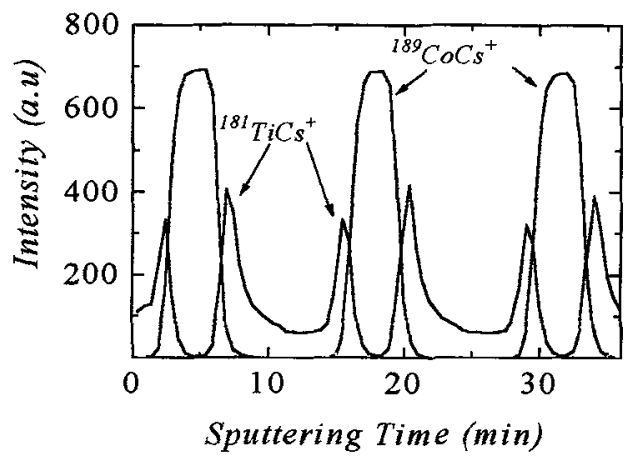

Figure 7 : Profil SIMS d'une multicouche $\mathrm{Co}-\mathrm{Ti}$ $\left[\mathrm{e}_{\mathrm{Co}}=\mathrm{e}_{\mathrm{Ti}}=300 \AA\right]$

concentration désirée. Les échantillons dont les résultats sont présentés sur les figures $10 \mathrm{a}$ et $\mathrm{b}$ sont constitués de couches de differents alliages d'épaisseurs comprises entre $3500 \AA$ et $1 \mu$ recouvertes d'une couche de $\mathrm{Ni}$ de $700 \AA$ puis d'une couche de $\mathrm{Ti}$ de $600 \AA$. Cet empilement tricouche permet une comparaison directe du signal $\mathrm{MCs}^{+}$dans l'alliage et dans le métal pur. La comparaison des intensités normalisées par rapport au métal pur montre que dans les alliages NiTi on observe une forte exaltation du rendement ionique de $\mathrm{TiCs}^{+}$(fig. 10a) par rapport au titane pur alors que parallèlement on observe une forte atténuation du signal $\mathrm{NiCs}^{+}$par rapport à celui du nickel pur (fig. 10b).

Si l'on represente en fonction des concentrations en nickel des alliages les intensités normalisées des signaux $\mathrm{TiCs}^{+}$et $\mathrm{NiCs}^{+}$on constate que si les signaux étaient proportionels aux concentrations on aurait une relation linéaire représentée par les droites dans les figures $11 \mathrm{a}$ et $11 \mathrm{~b}$, alors qu'en fait le signal $\mathrm{TiCs}^{+}$ est fortement exalté (d'un facteur 6 pour $65 \%$ atomique de $\mathrm{Ni}$ ) et celui de $\mathrm{NiCs}^{+}$décroit exponentiellement quand la concentration de Nickel diminue.

Si l'on examine sur la fig. 11a la courbe de reponse typique de $\mathrm{TiCs}^{+}$au niveau des interfaces dans les multicouches $\mathrm{Ni}-\mathrm{Ti}$ une correlation semble evidente entre la variation de composition dans la couche d'interdiffusion et l'effet d'exaltation observé dans les alliages en fonction de leur composition. En particulier le maximum du signal $\mathrm{TiCs}^{+}$observé sur le profil experimental (fig. 4) doit correspondre à une

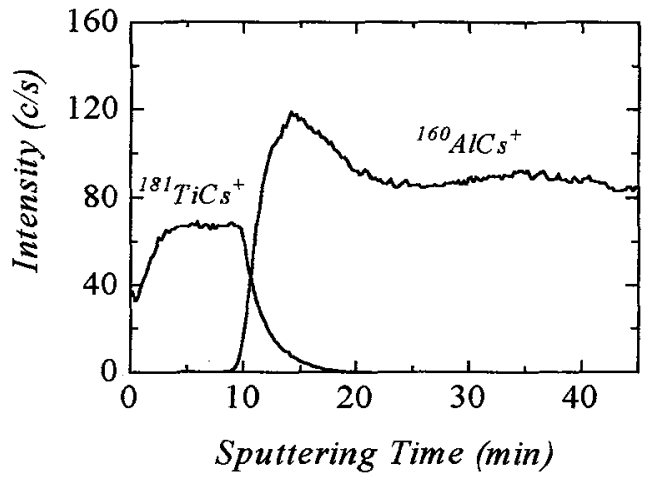

Figure 8: Profil SIMS d'une bicouche Ti-Al $\left[\mathrm{e}_{\mathrm{Ti}}=500 \AA ; \mathrm{e}_{\mathrm{Al}}=4000 \AA\right]$

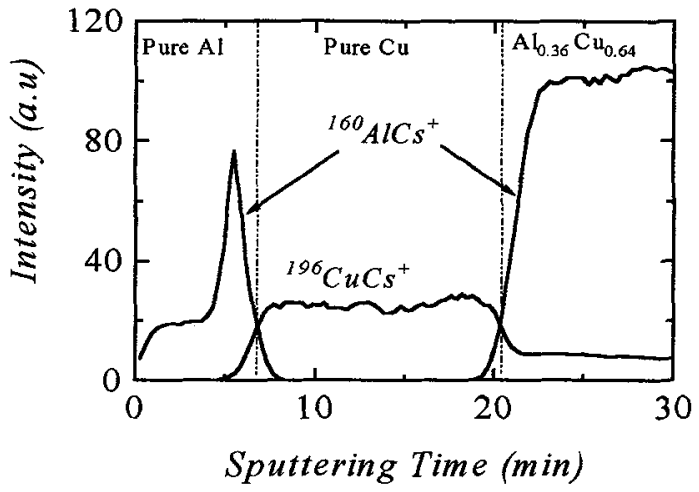

Figure 9: Profil SIMS d'une bicouche Al-Cu $\left[\mathrm{e}_{\mathrm{Al}}=600 \AA ; \mathrm{e}_{\mathrm{Cu}}=3000 \AA\right]$ déposée sur une couche d'alliage $\mathrm{Al}_{0,36} \mathrm{Cu}_{0,64}$ 


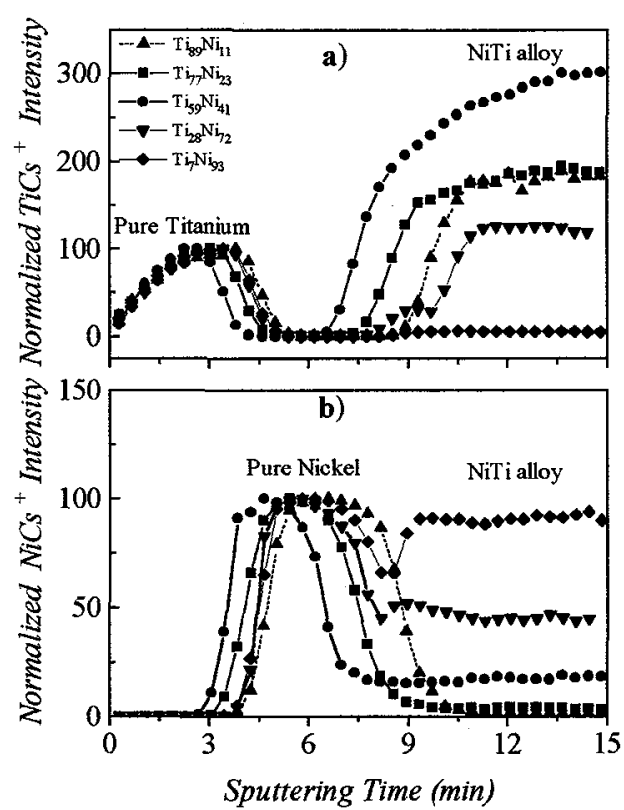

Figure 10: Profils SIMS de TiCs+ (a) et NiCs+ (b) donnés par différents alliages NiTi et normalisés par rapport aux métaux purs $\mathrm{Ni}$ et $\mathrm{Ti}$
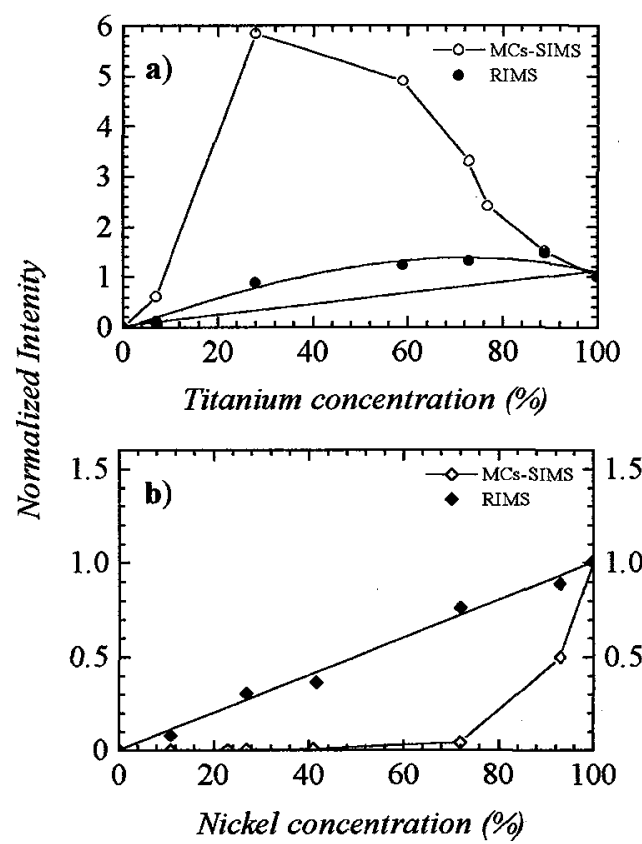

Figure 11: Comparaison des intensités normalisées, en fonction de la concentration des alliages, des ions moléculaires TiCs + et NiCs+ utilisés dans l'analyse SIMS, et des photoions $\mathrm{Ti}$ et Ni détectés après postionisation résonante (RIMS)

concentration en Ni proche de celle du maximum atteint (pour $65 \%$ at de $\mathrm{Ni}$ ) dans les courbes de réponse de l'ion $\mathrm{TiCs}^{+}$dans les alliages NiTi étudiés (fig.11a).

Un comportement similaire est illustré par la figure 9 qui représente le profil SIMS d'une couche d'alliage $\mathrm{Al}_{0,36} \mathrm{Cu}_{0,64}$ sur laquelle ont été déposées une couche de cuivre de $3000 \AA$ et une couche d'aluminium de $600 \AA$. On observe l'exaltation du signal de $\mathrm{AlCs}^{+}$à l'interface $\mathrm{Al}-\mathrm{Cu}$, ce qui est conforme aux conclusions précédentes puisque le rapport des densités $\mathrm{dCu} / \mathrm{dAl}=3.3$ est supérieur à la valeur limite de 1,4 . L'effet d'exaltation est également observé dans la couche d'alliage $\mathrm{Al}_{0,36} \mathrm{Cu}_{0,64}$ où le signal $\mathrm{AlCs}+$ est cinq fois plus intense que dans la couche d'aluminium pur.

Pour essayer de réduire les effets de matrice qui rendent très difficile l'interprétation quantitative des spectres SIMS nous avons utilisé la technique de post ionisation résonante (RIMS) mise au point au Laboratoire Phase du CNRS à Strasbourg [6]. Cette technique permet, grâce à un laser Nd-Yag couplé à un laser à colorant, de réaliser au voisinage de la surface une postionisation efficace des neutres pulvérisés qui sont majoritaires. On obtient alors pour le titane et le nickel des résultats plus proches de la linéarité en fonction des compositions étudiées. L'effet d'exaltation du titane est fortement atténué (on passe d'un facteur 6 à un facteur 1,5) (fig. 11a) et le Ni a un comportement quasi linéaire (fig. 11b).

Cet effet d'exaltation peut être utilisé pour révéler avec une excellente résolution la présence de couches d'interdiffusion d'épaisseur nanométrique. Les substrats doivent être parfaitement plans (poli spéculaire) et les interfaces très abruptes pour observer un effet d'exaltation important et contrasté. Ce phénomène est en effet rapidement atténué si l'on utilise des substrat rugueux ou si les interfaces deviennent diffuses par suite de phénomènes d'interdiffusion ou de mixing. Ceci est illustré par la fig.(12) relative à une multicouche FeTi soumise à un traitement de recuit. On observe une diffusion de l'hydrogène initialement fixé 


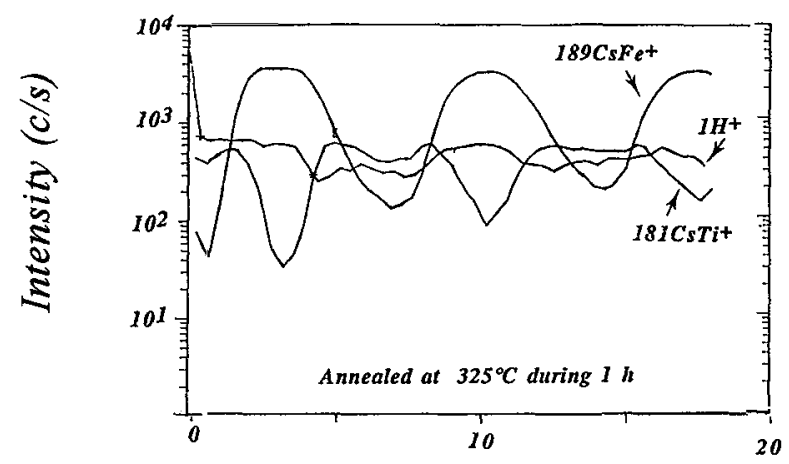

Sputtering Time (min)

Figure 12: Profil SIMS d'une multicouche Fe-Ti recuite à 325 pendant $1 \mathrm{~h}$

sélectivement sur le titane et une atténuation de l'amplitude de l'effet d'exaltation qui s'étale sur une profondeur plus importante du fait de l'interdiffusion des deux métaux.

Le mécanisme de formation des ions $\mathrm{MCs}^{+}$n'est pas complètement élucidé. Plusieurs études $[7,8]$ suggérant que ces ions moléculaires sont formés par la recombinaison au voisinage de la surface d'un atome neutre $\mathrm{M}$ pulvérisé et d'un ion $\mathrm{Cs}^{+}$repulverisé. La probabilité de recombinaison dépend de la concentration du Cs disponible en surface, concentration elle même liée à la quantité de Cs implanté et à la quantité qui migre à la surface par diffusion. Cette recombinaison dépend également du nombre d'atomes $\mathrm{M}$ pulvérisés (neutres ou ionisés) disponibles pour se recombiner avec $\mathrm{Cs}$ ou $\mathrm{Cs}^{+}$. Ce nombre résulte de cascades de collisions complexes qui dépendent fortement de la structure de la zone superficielle de la matrice.

Ce mécanisme complexe se traduit sur les profils SIMS par une variation importante de l'intensité du signal des ions secondaires $\mathrm{Cs}^{+}$dont le niveau baisse considérablement dans la zone correspondant à l'élément léger. Cette variation du niveau de $\mathrm{Cs}^{+}$est particulièrement visible sur les figures 1,2 et 5 relatives à des multicouches de V-Ti, Fe-V et Ni-V.

L'influence du rapport des densités est à rapprocher des calculs de Berg et coll. [9] qui montrent que la présence d'atomes lourds dans une matrice plus légère modifie la propagation des cascades de collisions et le transfert d'énergie aux atomes légers qui de ce fait peuvent se pulvériser ou s'ioniser plus facilement que dans le matériau pur. Ce phénomène localisé au niveau des interfaces peut modifier d'une façon importante les rendements de pulvérisation et d'ionisation.

\section{Références}

[1] Gao Y,. J. Appl. Phys. 64, (1988) 3760.

[2] Sella C., Nemraoui O., Maaza M., Miloche M., Kaabouchi M., in Secondary Ion Mass Spectrometry. SIMS IX. John Wiley Chichester, (1994) pp 887-890.

[3] Sella C., Maaza M., Miloche M., Kaabouchi M., Krishnan R., Surf. Coat. Technol. 60 (1993) 379384.

[4] Sella C., Miloche M., Nemraoui O., Kaabouchi M., Krishnan R., Maaza M. 10 $^{\text {th }}$ Conference on SIMS. Münster1-6 Oct 1995.

[5] Sella C., Kaabouchi M, Nemraoui O., Youn K. B., Maaza M, Surf. Coat. Technol. 74/75 (1995) 567.

[6] Kern P., Stuck R., Nemraoui O., Sella C., 10 ${ }^{\text {th }}$ Conference on SIMS. Münster, 1-6 Oct 1995.

[7] Wittmaak K., Nucl. Instrum. Methods B 64 (1992) 621-625.

[8] Gnaser H., Oechner H. Surf.Sci. Lett. 302 (1994) 1289.

[9] Berg S., Katardjiev I., Jonsson L., Hedlund C., Bäcklund Y., 4th International conference on Plasma Surface Eng., Garmisch-Partenkirchen, 19-23 Sept. 1994. 